\title{
Corrigendum to: "The effect of out of plane motion on 2D and 3D digital image correlation measurements" [Opt. Lasers Eng. 46(10)]
}

\author{
M.A. Sutton ${ }^{\mathrm{a}, *}$, J.H. Yan ${ }^{\mathrm{a}}$, V. Tiwari ${ }^{\mathrm{a}}$, H.W. Schreier ${ }^{\mathrm{b}}$, J.J. Orteu ${ }^{\mathrm{c}}$ \\ ${ }^{a}$ Department of Mechanical Engineering, University of South Carolina, 300 South Main Street, Columbia, SC 29208, USA \\ ${ }^{\mathrm{b}}$ Correlated Solutions Inc, 109 Kaminer Way, Columbia, SC, USA

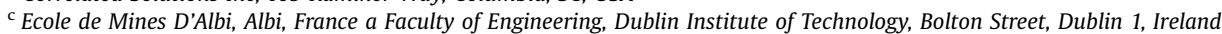

The authors regret that the following acknowledgements were missed in the original article:

The authors gratefully acknowledge the support of (a) the Army Research Office and Dr. Bruce Lamattina through W911NF 0610216 , (b) Dr. Stephen Smith and NASA Langley under Grant NASA NRA NNX07AB46A, (c) General Motors and Dr. Pablo Zavattieri through both contractual support ND0144200 and an unrestricted gift 002259249 and (d) the support provided by Dr. Jimmy Hsia and the National Science Foundation through NSF CMS 0556362. The technical assistance provided by Prof. Xiaomin Deng in the Department of Mechanical Engineering at the University of South Carolina and Dr. Hubert Schreier and Correlated Solutions Incorporated for supporting this effort through modifications to their software, VIC 2D, for our use is deeply appreciated. 